\title{
Manifestaciones neurológicas asociadas a COVID-19 en el Hospital Edgardo Rebagliati Martins, Perú
}

\author{
Neurological manifestations associated with COVID-19 in Edgardo Rebagliati Martins Hospital, Peru
}

Evelyn Mariños Sánchez 1,a, Poul Espino Alvarado ${ }^{1, \text { a }}$, Liliana Rodriguez 2, a, Elliot Barreto Acevedo ${ }^{2, \text { a }}$

\section{RESUMEN}

La enfermedad causada por el coronavirus 2 asociado al síndrome respiratorio agudo grave (SARS-CoV-2), se conoce formalmente como Coronavirus Disease 2019 (COVID-19). Su principal foco de infección es el sistema respiratorio, pero se han descrito también manifestaciones neurológicas. Objetivo: Describir las manifestaciones neurológicas en pacientes con COVID-19 en el hospital Edgardo Rebagliati Martins. Material y Métodos: Estudio observacional, descriptivo, transversal, empleando los registros médicos electrónicos de los pacientes hospitalizados con COVID -19 y dados de alta durante el periodo Abril-Agosto 2020. Se clasificaron a los pacientes como portadores de manifestaciones neurológicas del sistema nervioso central y periférico. Resultados: De 1122 pacientes, 354 (31,5\%) presentaron alguna manifestación neurológica, en tanto que 26,9\% mostraron síntomas del sistema nervioso central (SNC) y 8,4\% del sistema nervioso periféricos (SNP). El síntoma más frecuente del SNC fue cefalea $(19,7 \%)$ y como patologías: encefalopatía $(4,7 \%)$, el ataque cerebrovascular isquémico( $2,7 \%)$, crisis epiléptica sintomática aguda $(0,5 \%)$, hemorragia cerebral $(0,4 \%)$, encefalitis $(0,2 \%)$. Dentro de las manifestaciones del SNP predominaron mialgia (3,4\%), anosmia (3,1\%), disgeusia $(2,9 \%)$ y, como patologías, rabdomiólisis $(1,3 \%)$ y polirradiculoneuropatía aguda $(0,3 \%)$. La tasa de letalidad alcanzó el $32,7 \%$ con un OR de 1,4 . Conclusiones: Pacientes con infección por SARS-CoV-2 pueden presentar sintomatología neurológica como la descrita en el presente estudio, y sus manifestaciones deben ser consideradas en la atención oportuna e integral de los casos.

PALABRAS CLAVE: Manifestaciones neurológicas, COVID-19, SARS-CoV-2.

\section{SUMMARY}

The disease caused by coronavirus 2 associated with severe acute respiratory syndrome (SARS-CoV-2) is formally known as Coronavirus Disease 2019 (COVID-19). Its main focus is the respiratory system, but neurological manifestations have also been described. Objective: To describe the neurological manifestations in patients with COVID-19 at the Edgardo Rebagliati Martins hospital. Material and Methods: An observational, descriptive, crosssectional study, using the electronic medical records of COVID-19 hospitalized and discharged during the AprilAugust 2020 period. The patients were classified as carrying neurological manifestations of the central and peripheral nervous system. Results: Of 1122 patients, 354 (31.5\%) had some neurological manifestation while $26.9 \%$ showed symptoms of the central nervous system (CNS), and $8.4 \%$ of the peripheral nervous system (PNS). Among the CNS manifestations, the most frequent symptom was headache (19.7\%), and as pathologies: encephalopathy (4.7\%),

1 Departamento de Emergencia-CELIM, Hospital Nacional Edgardo Rebagliati Martins, EsSalud. Lima, Perú.

2 Departamento de Neurología, Hospital Nacional Edgardo Rebagliati Martins, EsSalud. Lima, Perú.

a Médico Asistente Neurólogo 
ischemic stroke $(2.7 \%)$, acute symptomatic seizure $(0.5 \%)$, cerebral hemorrhage $(0,4 \%)$, encephalitis $(0.2 \%)$. From the PNS manifestations, myalgia (3.4\%), anosmia (3.1\%) and dysgeusia (2.9\%) predominated and, as pathologies, rhabdomyolysis $(1.3 \%)$ and acute polyradiculoneuropathy $(0.3 \%)$. The fatality rate reached $32.7 \%$ with an OR of 1.4. Conclusions: Patients with SARS-CoV-2 infection can present neurological symptoms as those described in the present study, manifestations that should be considered in their timely and integrated care.

KEYWORDS: Neurological Manifestations, COVID-19, SARS-CoV-2.

\section{INTRODUCCIÓN}

La enfermedad causada por el coronavirus 2 asociado al síndrome respiratorio agudo grave (SARSCoV-2) se conoce como Coronavirus Disease 2019 (COVID-19); esta enfermedad es nueva en el mundo, y sus orígenes se remontan a diciembre del 2019 cuando en Wuhan, provincia de Hubei en China, se reportó los primeros casos con neumonía grave cuyo agente etiológico recién se descubrió en enero del $2020(1,2)$ China, was caused by a novel betacoronavirus, the 2019 novel coronavirus (2019-nCoV. En Perú, se confirmó la primera persona infectada por SARSCoV-2 el 6 de marzo de 2020. El 15 de agosto del 2020, la Universidad Johns Hopkins, de Estados Unidos, reveló que el Perú ocupó el primer puesto en muertes por millón de personas (651 fallecidos por millón) (3). El neurotropismo y el potencial papel neuropatógeno del SARS-CoV-2 se plantea en base a modelos en animales y casos de afección neurológica asociada a infección por otros coronavirus (SARS-CoV, MERS CoV, HCoV-OC43). El SARS-CoV-2 a través de su proteína $\mathrm{S}$ se fija al receptor de la enzima convertidora de angiotensina 2 (ECA-2) para invadir las células y replicarse. Los mecanismos que explican el neurotropismo del SARS-CoV-2, describen la identificación de ARNm de ACE-2 y células doblemente positivas a ACE-2 y la proteasa transmembrana serina 2 (TMPRSS2), entre otras, en neuronas y células gliales, en la corteza cerebral, el cuerpo estriado, el hipotálamo, la sustancia negra y el tallo cerebral, lo que confiere al SNC ser un objetivo directo del SARS-CoV-2. Varios estudios explican los mecanismos neurotrópicos del SARS-CoV-2 como posibles rutas de acceso viral al cerebro, como la ruta transcribial (describe una infección del epitelio olfatorio y transmisión sucesiva a través de la placa cribiforme a el espacio subaracnoideo, vía implicada en la anosmia), el transporte axonal y transferencia transsináptica (infección de varias terminales nerviosas periféricas y una extensión a lo largo de neuronas, como el bulbo olfatorio, el nervio trigémino o el nervio vago en el tracto respiratorio o gastrointestinal), y la ruta hematógena y / o linfática (migración a través del endotelio cerebral por la infección directa de células endoteliales de la microvasculatura cerebral o por endocitosis, a través de leucocitos infectados por virus o alteración de las estrechas uniones de las células endoteliales de la microvasculatura endotelial). No toda las manifestaciones neurológicos requieren una infección directa del SNC, la neurotoxicidad indirecta puede resultar secundaria a patogénesis inmunomediada (anticuerpos, citoquinas), disfunción de la coagulación, entre otros (4-9).

La primera serie que describe manifestaciones neurológicas en pacientes con COVID-19 fue la publicada por Mao et al. en China, quién reportó que 78 de 214 pacientes presentaron síntomas neurológicos, las clasificaron en 3 categorías: manifestaciones del sistema nervioso central, periférico y del músculo esquelético(10).

La sociedad española de neurología (SEN), notificaron 131 eventos neurológicos, correspondientes a 16 categorías de síndrome neurológicos, siendo las más frecuentes: el síndrome confusional o encefalopatía leve a moderada $(28,3 \%)$, infarto cerebral $(22,8 \%)$, la anosmia/hiposmia $(19,6 \%)$, la cefalea $(14,1 \%)$, crisis sintomática aguda $(12 \%)$, polirradiculoneuropatía $(7,6 \%)$, hemorragia cerebral $(4,3 \%)$, neuropatía oculomotora $(3,3 \%)$, encefalitis $(2,2 \%)$ y otros $(11)$.

Hay estudios previos que describen las características clínicas de pacientes con COVID-19 en nuestro medio, pero ninguna de forma específica sobre sus manifestaciones neurológicas, salvo algunos reportes de casos publicados de encefalitis y accidente cerebrovascular isquémico asociado a COVID-19 por los presentes autores $(12,13)$.

Se realizó el presente trabajo con el objeto de describir las manifestaciones neurológicas en pacientes con COVID-19 en un Hospital Terciario peruano. 


\section{MATERIAL Y MÉTODOS}

Se realizó un estudio observacional, descriptivo, retrospectivo de corte transversal desde el periodo de abril a agosto del 2020, empleando los registros médicos electrónicos de los egresos, se consideraron aquellos con los diagnósticos según el CIE-10 U07.1 (diagnóstico de coronavirus identificado por Reacción en cadena de polimerasa en tiempo real de hisopado nasofaríngeo (RT- PCR ) o Prueba rápida) o CIE-10 U07.2 (sospechoso de coronavirus por patrón radiográfico o tomográfico). Según fuente del Sistema Informático se contaron 4535 pacientes con infección por SARS-CoV-2. Se realizó una selección no probabilística, obteniéndose una muestra de 1122 , que representa el $24,74 \%$.

La evaluación de los pacientes con patologías neurológicas fue realizada por el neurólogo de turno y el seguimiento de los casos por el equipo de neurólogos que conforman el estudio de investigación.

Como criterios de inclusión se consideraron: pacientes mayores de 18 años; manifestaciones neurológicas: del SNC: cefalea, ataque cerebrovascular isquémico o hemorrágico, encefalitis, meningoencefalitis, crisis sintomática aguda, encefalopatías, otros (mielitis, cuadro extrapiramidal); del sistema nervioso Periférico (SNP): compromiso de pares craneales (anosmia, ageusia/hipogeusia, otros), polirradiculoneuropatía (síndrome de Guillain barré), musculoesqueléticas (síntomas mialgias y patologías miopatías, rabdomiólisis, otros). Criterios de exclusión: antecedentes de accidentes cerebrovasculares isquémicos cuya sintomatología focal fue similar a las lesiones previas, pacientes con accidente cerebrovascular hemorrágico de etiología aneurismática, malformación vascular o contusión hemorrágica, pacientes con secuelas neurológicas previas tipo: enfermedades neuromusculares, miopatías, esclerosis lateral amiotrófica (si se desea evaluar sintomatología musculoesquelética).

Las variables categóricas cualitativas y cuantitativas, serán presentadas como frecuencias con sus respectivos porcentajes, media, mediana, rango, rango intercuartílico, según corresponda usando SPSS versión 25.0. Esta investigación cuenta con la aprobación del Comité de ética según resolución $\mathrm{N}^{\circ}$ 42-IETSI-ESSALUD-2020.

\section{RESULTADOS}

El estudio contó con 1122 egresados por infección por SARS-CoV-2, siendo la edad promedio 58 años, 795 fueron mayores de 50 años (71\%); 767 de sexo masculino (68\%); las comorbilidades más frecuentes: hipertensión arterial 317 (28\%), diabetes mellitus 204 (18\%), obesidad $180(16 \%)$; con un tiempo de enfermedad $\leq 7$ días $682(60 \%)$; los síntomas de presentación: disnea $867(77 \%)$, tos $675(60 \%)$, fiebre 526 (46\%), malestar general 370 (32\%), cefalea $222(20 \%)$; síndromes clínicos de gravedad al ingreso a la emergencia: neumonía severa 580 (51\%), síndrome de distrés respiratorio del adulto 286 (25\%); tratamiento empleado azitromicina 571(51\%), hidroxicloroquina 352 (31\%), dexametasona 346 (30\%), metilprednisolona 280 (25\%); complicaciones: síndrome de distrés respiratorio del adulto $286(25 \%)$, sepsis 169 (15\%), metabólicas $141(12,5 \%)$, injuria renal aguda $89(7,9 \%)$; personal de salud hospitalizado $71(6,3 \%)$, fallecidos 314 (27,9\%). Exámenes paraclínicos, se realizó prueba molecular RT-PCR positivo en 564, Prueba rápida reactiva en 511, Radiografía de tórax sugestiva en 169, Tomografía de Tórax con patrón sugestivo para infección por SARSCoV-2 en 943, CO-RADS 5-6 874 (92\%); PaO2/ $\mathrm{FiO} 2<300$ 771(69\%) (tabla1).

De los 1122 casos de pacientes con infección por SARS-CoV-2, 354(31,5\%) presentó alguna manifestación neurológica. La mediana de edad fue de 58años, 242 mayores de 50años (68\%); sexo masculino $231(65,2 \%)$; las comorbilidades en orden de frecuencia: hipertensión arterial $102(29 \%)$, diabetes mellitus $70(20 \%)$, obesidad 49 (14\%), enfermedad renal crónica 24 (7\%), enfermedad cardiaca $19(5,4 \%)$. Las manifestaciones del SNC fueron 302 (26,9\%) y SNP $94(8,4 \%)$. Dentro de las manifestaciones neurológicas del SNC, el síntoma más frecuente fue la cefalea $222(19,7 \%)$ y como patologías: encefalopatía $53(4,7 \%)$, ataque cerebrovascular (ACV) isquémico $30(2,7 \%)$, crisis sintomáticas agudas $6(0,5 \%)$, ACV hemorrágico $4(0,4 \%)$, encefalitis $2(0,2 \%)$, parkinsonismo $2(0,2 \%)$, meningoencefalitis viral 1 $(0,1 \%)$. Dentro de las manifestaciones neurológicas del SNP: como síntomas mialgia $38(3,4 \%)$, anosmia 35 $(3,1 \%)$, ageusia/disgeusia $32(2,9 \%)$ y como patologías rabdomiólisis $15(1,3 \%)$, polirradiculoneuropatía aguda $3(0,3 \%)$ (tabla 2$)$.

\section{Manifestaciones del SNC}

- Cefalea, es el síntoma neurológico más frecuente, predominó en el sexo masculino (66\%), la mediana 
Tabla 1. Características clínicas - paraclínicas de pacientes con infección por SARS-CoV-2, HNERM, Perú

\begin{tabular}{|c|c|c|c|c|c|}
\hline EDAD & $\mathbf{n}$ & $\%$ & SÍNDROMES CLÍNICOS DE GRAVEDAD & $\mathbf{n}$ & $\%$ \\
\hline $58,6(18-99)^{*}$ & & & Infección no complicada & 63 & 5,6 \\
\hline $18-30$ & 43 & 4 & Neumonía leve & 138 & 12,3 \\
\hline $31-40$ & 101 & 9 & Neumonía severa & 580 & 51,6 \\
\hline $41-50$ & 183 & 16 & SDRA & 286 & 25,4 \\
\hline $51-60$ & 276 & 25 & Sepsis & 27 & 2,4 \\
\hline $61-70$ & 266 & 24 & Shock séptico & 25 & 2,2 \\
\hline $71-80$ & 168 & 15 & PERSONAL DE SALUD & 71 & 6,3 \\
\hline$>=81$ & 85 & 7 & TRATAMIENTO & & \\
\hline SEXO & & & Hidroxicloroquina & 352 & 31 \\
\hline Masculino & 767 & 68 & Azitromicina & 571 & 51 \\
\hline COMORBILIDADES & & & Ivermectina & 115 & 10 \\
\hline Hipertensión arterial & 317 & 28 & Metilprednisolona & 280 & 25 \\
\hline Diabetes mellitus & 204 & 18 & Dexametasona & 346 & 30 \\
\hline Obesidad & 180 & 16 & COMPLICACIONES & & \\
\hline Enf. Renal Crónica & 68 & 6 & SDRA Severo & 286 & 25,4 \\
\hline Enf. Cardiaca & 60 & 5 & Sepsis & 169 & 15 \\
\hline Enf Neurológicas & 44 & 4 & Metabólicas & 141 & 12,5 \\
\hline Asma & 40 & 3,5 & Injuria renal aguda & 89 & 7,9 \\
\hline Neoplasia/QT-RT & 36 & 3 & Neurológicas & 45 & 4 \\
\hline Otras & 159 & 12 & Otras & 95 & 8,3 \\
\hline Ninguna & 360 & 32 & VENTILACIÓN MECÁNICA & 193 & 17,2 \\
\hline TIEMPO DE ENFERMEDAD & & & Unidad de Cuidados Intensivos & 116 & 10,3 \\
\hline$<=7$ días & 682 & 60 & FALLECIDOS & 314 & 27,9 \\
\hline 8 a 14 & 349 & 32 & \multicolumn{3}{|c|}{ EXÁMENES DE PARACLÍNICOS LABORATORIO } \\
\hline$>=15$ & 89 & 8 & RT-PCR POSITIVO & 564 & \\
\hline \multicolumn{3}{|c|}{ SÍNTOMAS DE PRESENTACIÓN DEL SARSCOV2 } & Prueba rápida reactiva & 511 & \\
\hline Disnea & 867 & 77 & Radiografia sugestiva & 169 & \\
\hline Tos & 675 & 60 & TEM Tórax & 943 & \\
\hline Fiebre & 526 & 46 & CO-RADS 3-4 & 57 & 6 \\
\hline Malestar general & 370 & 32 & CO-RADS 5-6 & 874 & 92 \\
\hline Cefalea & 222 & 20 & $\mathrm{~Pa} 02 / \mathrm{Fi} 02<100$ & 212 & 19 \\
\hline Odinofagia & 217 & 19 & $\mathrm{~Pa} 02 / \mathrm{Fi} 02 \quad 101-200$ & 228 & 20 \\
\hline Diarrea & 73 & 6 & $\mathrm{~Pa} 02 / \mathrm{Fi} 02 \quad 201-300$ & 331 & 30 \\
\hline Mialgia & 38 & 3 & $\mathrm{~Pa} 02 / \mathrm{Fi} 02>301$ & 342 & 31 \\
\hline Anosmia & 35 & 3 & & & \\
\hline Disgeusia/Ageusia & 32 & 2 & & & \\
\hline Otros & 141 & 11 & & & \\
\hline
\end{tabular}

Mediana-Rango* RT-PCR+: Reacción en cadena de polimerasa en tiempo real de hisopado Nasofaríngeo.

HNERM: Hospital Nacional Edgardo Rebagliati Martins

de edad fue 56años (rango 18-86años), con una mediana de 8días desde el inicio de enfermedad, 63 $(28 \%)$ de fallecidos.

- Encefalopatía: es la segunda manifestación neurológica, la mediana de edad fue 70 años, $64 \%$ sexo masculino, el $49 \%$ presentó síndrome de distrés respiratorio agudo, con una mediana de 7días desde el inicio de enfermedad, 38 (71\%) de fallecidos.

- ACV Isquémico fueron 30 casos, representó el $88 \%$ del total de ACV, el $2,7 \%$ del total de la muestra de pacientes infectados por SARS-CoV-2 
Tabla 2. Características clínicas de pacientes con manifestaciones neurológicas con infección por SARS-cov-2, HNERM, Perú

\begin{tabular}{lcc}
\hline \multicolumn{1}{c}{ EDAD (años) } & N & \% \\
\hline 58 (18-99) * & & 3 \\
$18-30$ & 10 & 8 \\
$31-40$ & 27 & 21 \\
$41-50$ & 75 & 25 \\
$51-60$ & 89 & 21 \\
$61-70$ & 76 & 13 \\
$71-80$ & 44 & 9 \\
$>=81$ & 33 & \\
SEXO & & 65,2 \\
Masculino & 231 & 29 \\
COMORBILIDADES & & 20 \\
Hipertensión arterial & 102 & 14 \\
Diabetes mellitus & 70 & 7 \\
Obesidad & 49 & 5,4 \\
ENF. Renal Crónica & 24 & 3 \\
Enfermedad Cardiaca & 19 & 18 \\
Asma & 11 & 35 \\
Otras & 63 & \\
Ninguna & 121 &
\end{tabular}

MANIFESTACIONES NEUROLÓGICAS

\begin{tabular}{lccc} 
& & $\begin{array}{c}\text { Infección por } \\
\text { SARS-CoV-2 }\end{array}$ & $\begin{array}{l}\text { Pacientes con Manifestaciones } \\
\text { Neurológicas asociadas a Infección por } \\
\text { SARS-CoV-2 } \\
\mathbf{n = 3 5 4}\end{array}$ \\
\hline \multicolumn{1}{c}{ SISTEMA NERVIOSO CENTRAL (302) } & N=1122 & $\mathbf{\%}$ & $\mathbf{\%}$ \\
\hline SINTOMAS & 222 & 19,7 & 62,7 \\
Cefalea & & & \\
PATOLOGIA & 53 & 4,7 & 14,9 \\
Encefalopatía & 30 & 2,7 & 8,5 \\
ACV isquémico & 6 & 0,5 & 1,7 \\
Crisis Sintomática Aguda & 4 & 0,4 & 1,1 \\
ACV hemorrágico & 2 & 0,2 & 0,6 \\
Encefalitis & 2 & 0,2 & 0,6 \\
Parkinsonismo & 1 & 0,1 & 0,3 \\
Meningoencefalitis & & & \\
SISTEMA NERVIOSO PERIFÉRICO (94) & & & 9,7 \\
SINTOMAS & & 3,4 & 9 \\
Mialgia & 38 & 3,1 & \\
Anosmia & 35 & 2,9 & 4,2 \\
Disgeusia/Ageusia & 32 & & 1,2 \\
PATOLOGIA & & 1,3 & 33 \\
Rabdomiolísis & 15 & 0,3 & \\
Polirradiculoneuropatia aguda & 3 & 10 & \\
Fallecidos & 116 & & \\
\hline
\end{tabular}

Mediana-Rango* ACV: Ataque cerebrovascular 
y el $8,6 \%$ de las manifestaciones neurológicas, la mediana para la edad fue 69 años, siendo el $81 \%$ mayores de 50 años; sin embargo 6 eventos se dieron en pacientes adultos jóvenes (menores de 50 años) el de menor edad tuvo 38 años, el sexo masculino fue un 50\%. El 86,6\% tuvo al menos un factor de riesgo conocido para enfermedad cerebro vascular como hipertensión arterial $(50 \%)$, enfermedad cardiaca $(30 \%)$, diabetes mellitus (26\%), obesidad (16\%); ingresaron con un tiempo de enfermedad menor de 24 horas $(56 \%)$, con síntomas concomitantes con infección por SARS-CoV-2 como disnea (53\%), desarrollaron neumonía severa (76\%). El 93\% tuvo un ACV

Tabla 3. Características clínicas de pacientes con ataque cerebrovascular asociados a infección por SARS-CoVS2, HNERM, Perú

\begin{tabular}{|c|c|c|c|c|c|}
\hline & $\mathbf{N}^{\circ}$ & $\%$ & \multicolumn{3}{|c|}{ Escala de NIHSS } \\
\hline ACV & 34 & & Leve $<4$ & 2 & 7 \\
\hline Isquémico & 30 & 88 & Moderado $<16$ & 15 & 50 \\
\hline Hemorrágico & 4 & 12 & Grave $<25$ & 11 & 36 \\
\hline \multicolumn{3}{|l|}{ ACV ISQUÉMICOS } & Muy grave $\geq 25$ & 2 & 7 \\
\hline \multicolumn{3}{|l|}{ Edad (años) } & \multicolumn{3}{|c|}{ Territorio Vascular } \\
\hline Mediana & 69 & & $\mathrm{ACM}$ & 18 & 60 \\
\hline $18-30$ & 0 & 0 & ACA & 2 & 7 \\
\hline $31-40$ & 1 & 3 & $\mathrm{ACP}$ & 3 & 10 \\
\hline $41-50$ & 5 & 17 & AV & 4 & 13 \\
\hline $51-60$ & 1 & 3 & $\mathrm{AB}$ & 1 & 4 \\
\hline $61-70$ & 8 & 27 & Multinfarto & 2 & 6 \\
\hline $71-80$ & 7 & 24 & \multicolumn{3}{|l|}{ Lado Afectado } \\
\hline$>=81$ & 8 & 27 & Izquierda & 13 & 43 \\
\hline SEXO & & & Derecha & 15 & 50 \\
\hline Masculino & 15 & 50 & Ambas & 2 & 7 \\
\hline \multicolumn{3}{|l|}{ COMORBILIDADES } & \multicolumn{3}{|c|}{ Tamaño del ACV } \\
\hline Hipertensión arterial & 15 & 50 & Lacunar & 5 & 16 \\
\hline Enf. Cardiaca & 9 & 30 & Mediano & 8 & 27 \\
\hline Diabetes mellitus & 8 & 26 & Extenso & 17 & 57 \\
\hline Obesidad & 5 & 16 & \multicolumn{3}{|l|}{ Tratamiento } \\
\hline Enf. Renal Crónica & 3 & 10 & Antiagregación & 24 & 80 \\
\hline Neoplasia/QT-RT & 2 & 6 & Anticoagulación & 2 & 7 \\
\hline Otros & 2 & 6 & Trombolisis & 1 & 3 \\
\hline Ninguno & 4 & 13 & \multicolumn{3}{|c|}{ Síntomas de presentación de COVID } \\
\hline \multicolumn{3}{|c|}{ TE desde el inicio de síntomas de ACV(horas) } & Disnea & 16 & 53 \\
\hline$<4.5$ & 3 & 10 & Malestar general & 12 & 40 \\
\hline $4.5-24$ & 12 & 40 & Tos & 9 & 30 \\
\hline$>24 h-48$ & 3 & 10 & Fiebre & 7 & 23 \\
\hline$>48 \mathrm{~h}-72$ & 6 & 20 & Fallecidos & 10 & 33 \\
\hline$>72$ & 6 & 20 & & & \\
\hline
\end{tabular}

ACV: Ataque cerebrovascular TE: tiempo de enfermedad. NIHSS: National institute of Health Stroke Scale. ACM: arteria cerebral media. ACA: arteria cerebral anterior. ACP: arteria cerebral posterior. AV: arteria vertebral. AB: arteria basilar. 
Manifestaciones neurológicas asociadas a COVID-19 en el Hospital Edgardo Rebagliati Martins, Perú

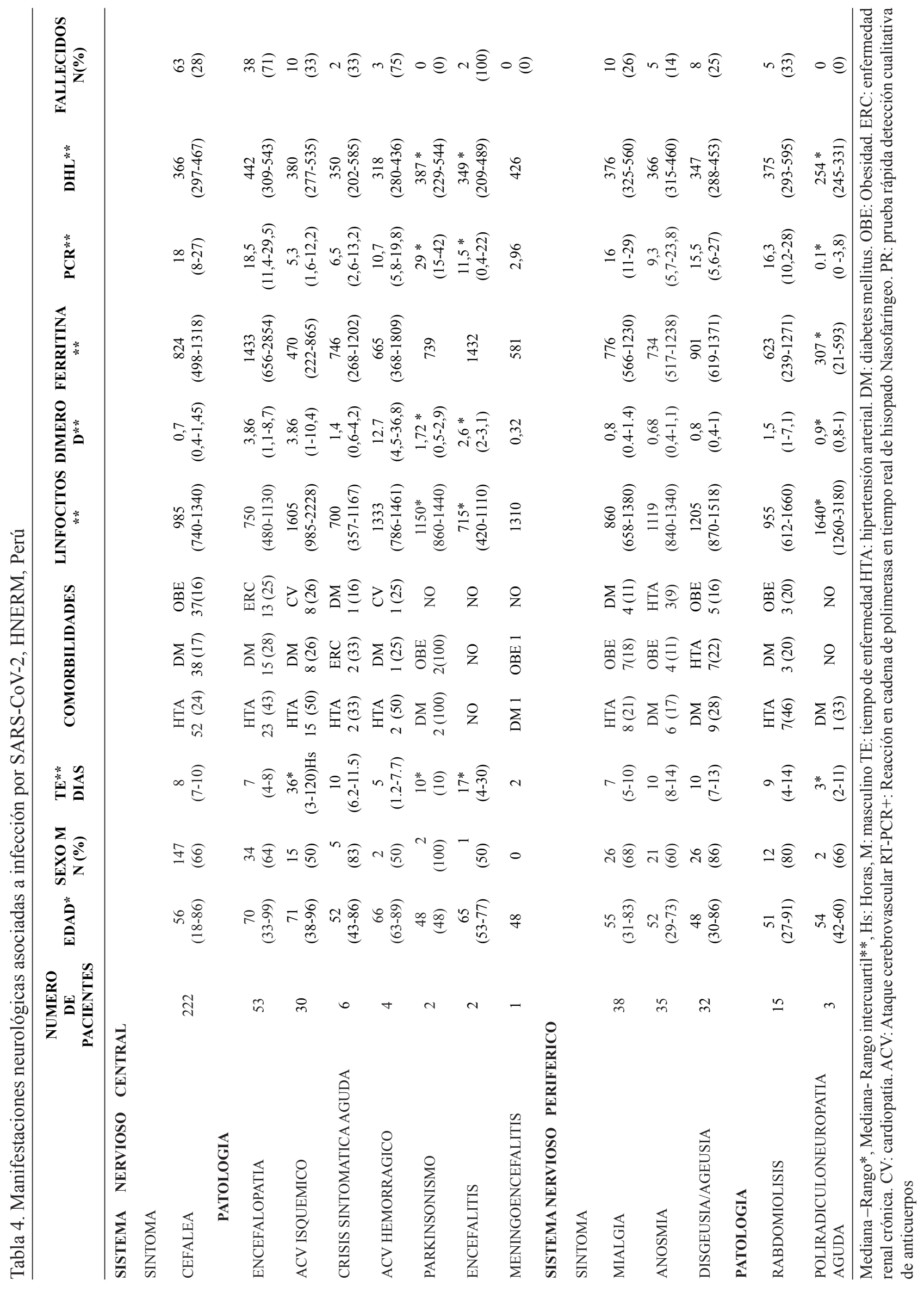


isquémico moderado a severo según la National institute of Health Stroke Scale (NIHSS), el subgrupo con puntaje de NIHSS de $5 \mathrm{a}<16$ fue de $50 \%$, tamaño del $\mathrm{ACV}$ isquémico extenso (57\%). El territorio vascular más afectado fue la arteria cerebral media (60\%), lado derecho $(50 \%)$, tratamiento principalmente antiagregación $(80 \%)$, el 3\% recibió trombolisis, fallecidos $10(33 \%)$. Los marcadores protrombóticos estuvieron elevados, dímero $\mathrm{D}$ con una mediana 3,86 y fibrinógeno 488,5 (tabla 3).

- Crisis sintomáticas agudas: la mediana de edad fue 52 años, sexo masculino $50 \%$, con una mediana de 10 días desde el inicio de enfermedad, cuadro clínico de neumonía severa en $66 \%$, fallecidos 2 $(33 \%)$.

- Ataque cerebrovascular hemorrágico: fueron 4 casos ( $12 \%$ del total de ACV), $0,4 \%$ de la muestra de pacientes con infección por SARS-CoV-2, la mediana de edad 66años, sexo masculino $50 \%$, con un tiempo medio de enfermedad de 5 días, cuadro clínico de neumonía severa en $50 \%$, fallecidos 3 (75\%).

\section{Manifestaciones del SNP}

- Mialgia: es el síntoma neurológico del SNP por afectación musculoesquelética más frecuente, 38 casos (3,4\%), la mediana de edad 55años, sexo masculino $68 \%$, con una mediana de 7 días desde el inicio de enfermedad, fallecidos10 (26\%).

- Anosmia, 35 pacientes (3,1\%) de las manifestaciones del SNP, la mediana de edad 52 años, sexo masculino $60 \%$, con una mediana de 10 días desde el inicio de enfermedad, cuadro clínico de neumonía severa en 57\%. fallecidos 5 (14\%).

- Ageusia/Disgeusia, 32 casos (8,8\%), la mediana de edad 48años, sexo masculino $86 \%$, con una mediana de 10 días de enfermedad, cuadro clínico de neumonía severa en 53\%, fallecidos $8(25 \%)$.

- Rabdomiólisis: se encontró 15 casos $(1,3 \%)$, la mediana de edad 51años, sexo masculino $80 \%$, con una mediana de 9 días de enfermedad, CPK 793 (489-4659), fallecidos 5 (33\%).

- Polirradiculoneuropatía aguda: evaluamos 3 casos $(0,3 \%)$, la mediana de edad 54años, sexo masculino $66 \%$, con una mediana de 3 días desde el inicio de síntomas (rango de 2 a 11 días), cuadro clínico de neumonía severa en $66 \%$. RT-PCR fue positiva en 1 de 2, prueba rápida reactiva en los 3 casos, tomografía de tórax con CO-RADS 5-6 en $100 \%$. Los 3 casos con cuadriparesia arrefléxica aguda ascendente, sin nivel sensitivo, ni trastornos esfinteriano, 2 de ellos con disautonomías, 2 con estudio de LCR con disociación albúmino citológica, a uno de ellos no se efectivizóla punción lumbar por plaquetopenia severa, debido a Púrpura trombocitopénica idiopática refractaria a corticoides. Recibieron inmunoglobulinas. A ninguno se les realizó estudio de electromiografía.

Hallazgos de laboratorioLos pacientes con manifestaciones neurológicas, presentaron medianas con tendencia a linfopenia e incremento de marcadores inflamatorios como dímero D, ferritina, Proteína $\mathrm{C}$ reactiva (PCR), DHL consideradas como factores de mal pronóstico (tabla 4).

\section{DISCUSIÓN}

Varios estudios han mostrado pacientes con infección por SARS-CoV-2 con manifestaciones neurológicas. La primera descripción fue realizada en la ciudad de Wuhan, China, por Ling Mao et al. las clasificaron en 3 categorías: manifestaciones del SNC (mareos, cefalea, alteración de conciencia, enfermedad cerebrovascular aguda, ataxia y convulsiones), manifestaciones del SNP (alteraciones del gusto, del olfato, de la visión) y manifestaciones de lesiones de músculo esquelético. En dicha serie el $36 \%$ presentó alguna manifestación neurológica, porcentaje similar encontrada en el presente estudio (31,5\%). En el estudio de Mao, la edad media fue de 52,7 años, 58 años en el presente estudio y 62,5 años para la SEN. En relación al género, en el estudio de Mao 40,7\% eran masculino, en el nuestro $65,2 \%$, similar al estudio de la SEN $60 \%$. La hipertensión y la diabetes fueron las comorbilidades más frecuentes para los tres estudios, en nuestro estudio la tercera comorbilidad fue la obesidad con un $14 \%$. Los síntomas más comunes descritos por Mao al inicio de la enfermedad fueron fiebre $(61,7 \%)$, tos $(50 \%)$ y anorexia $(31,8 \%)$, en nuestra revisión: disnea, tos y fiebre $(10,11)$.

En nuestro estudio las manifestaciones más frecuentes se dieron a nivel del SNC 302 pacientes $(26,9 \%)$ datos similares a los publicados por Mao 53 (24\%). Dentro de estas manifestaciones destaca la cefalea (19\%), a diferencia de la serie de Mao, dónde predominaron los mareos $(16,8 \%)$ y en segundo lugar la cefalea $(13,1 \%)$, para la SEN ocupó el cuarto lugar con un $14,1 \%$. En nuestra serie no se describió mareos. La segunda manifestación encontrada fue la encefalopatía (4,8\%), a diferencia del SEN, el cuadro confusional y encefalopatía $(28,3 \%)$ fue su primera manifestación (10). Helms et al. reportó 


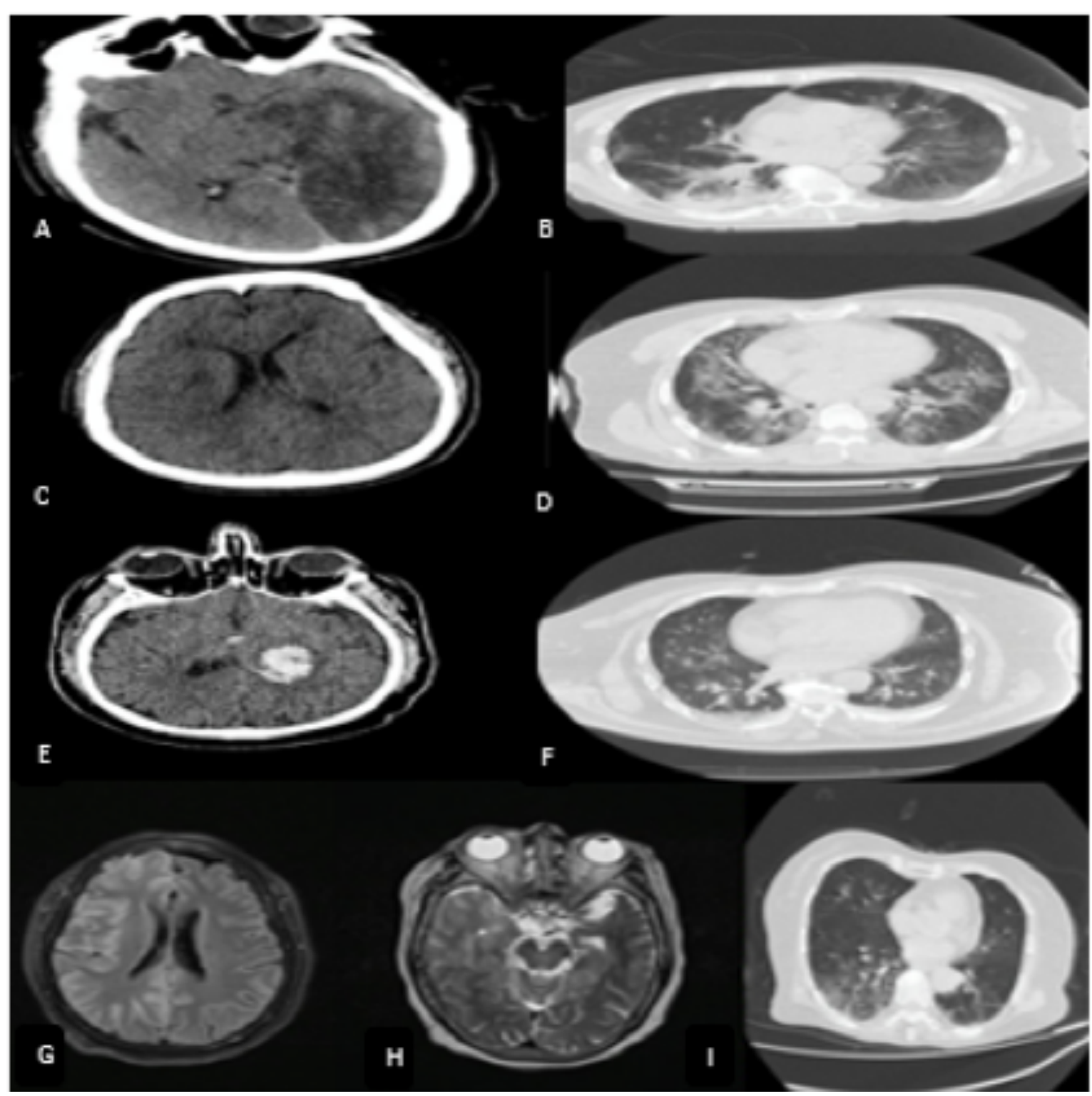

Figura 1. Infarto cerebral de vaso grande en región occipito temporal izquierda en paciente adulto mayor con neumonía por SARS-CoV-2 sin otro factor de riesgo vascular conocido (A,B). Infarto lacunar para ventricular derecho en paciente adulto joven con neumonía por SARS-CoV-2 sin factor de riesgo vascular conocido (C,D). Hemorragia intracerebral en ganglios basales izquierdos en paciente con neumonía por SARS-CoV-2 (E,F). RM de encéfalo con hiperseñal en FLAIR y T2, giriforme a nivel cortical y extensión a zonas subcorticales que afectan la región temporal, frontal y parietal derecha compatible con encefalitis en paciente con neumonía por SARS-CoV-2 (G,H,I).

compromiso neurológico en el $84 \%$ de los pacientes con infección severa por SARS-CoV-2 sus principales manifestaciones fueron encefalopatía, agitación, confusión (14). El 49\% de nuestra serie presentó síndrome de distrés respiratorio agudo severo del adulto, la encefalopatía puede estar en relación a inflamación sistémica,hipoxemia, alteración metabólica, polifarmacia y otros factores (15).
En relación al ACV Isquémico, el porcentaje fue de $2,7 \%$, en comparación a otros reportes de países europeos (Italia, Francia y Holanda), 1 de Asia (china) y 1 de América, donde describen entre 0,9\% a 2,7\%(10,16-20). La mediana para la edad fue 69 años, estos datos no están lejos de lo publicado en una reciente revisión sistemática que incluye 135 pacientes (promedio de 63 años)(19); además casos en adultos jóvenes también han sido descritos $(20,21)$ al igual que 
en nuestro estudio(20,21). La mayoría de los pacientes de nuestro estudio (93\%) tuvieron un ACV isquémico moderado a severo según el puntaje de NIHSS, que estaría en relación con lo publicado recientemente, en donde se describe en un análisis comparativo, que los pacientes con COVID-19 sufrieron ACV más graves, con una puntuación NIHSS más alta y una mayor mortalidad hospitalaria, a diferencia de los pacientes COVID-19 negativos o controles históricos. El 3\% recibió trombólisis, cifra que resulta ser baja comparado con lo reportado en otros estudios que alcanza el $33,7 \%$.Además la mortalidad en este patología es alta, en nuestro estudio fue $33 \%$ similar a lo descrito en una revisión sistemática que alcanza el 38\% (16,19-21). La concurrencia entre ACV isquémico e infección por SARS-CoV-2 se ha ido reportando con el pasar de los meses desde que inicio la pandemia, si bien es cierto ambas entidades nosológicas comparten factores de riesgo, la infección por SARS-CoV-2 podría ser un desencadenante, se postulan diversos mecanismos de causalidad, tanto de forma directa e indirecta. La forma directa sería a través del daño de células endoteliales de los vasos arteriales cerebrales pequeños (produciendo infartos lacunares) o vasos de mayor calibre (ocasionando disecciones y por ende infartos cerebrales); el mecanismo indirecto se explica por el estado protrombótico (documentado por el aumento del dímero $\mathrm{D}$, fibrinógeno), estado hiperinflamatorio y además un posible daño miocárdico que condicionaría eventos cardioembolígenos(12,22-24). Hubo limitaciones en la realización de los exámenes de apoyo diagnóstico (Figura 1).

En la SEN reportaron crisis epilépticas (12\%; 4 de ellos $(4,3 \%)$ con status y 6 de ellos sin antecedentes de crisis) (10), en nuestro estudio representan el 1,7\% de los pacientes con manifestaciones neurológicas. Pueden atribuirse a complicaciones como la hipoxemia, trastornos hidroelectrolíticos, descompensaciones de comorbilidades como diabetes, hipertensión, infección sin evidencia de encefalitis, entre otras. Una limitante fue que en algunos casos de crisis sutiles o estatus no convulsivos (pacientes en ventilación mecánica), no se pudo determinar por no contar con equipo de electroencefalograma en zona COVID.

Los casos de ACV hemorrágicos a diferencia de los ACV isquémicos han sido menos reportados en pacientes con COVID-19. En nuestra revisión fueron 4 casos. La SEN reportó 4 (4,3\%); Mao, describió 1 paciente; Li reportó 1 caso de un total de 11 pacientes(25)acute cerebrovascular disease (CVD,
Sharifi 1 caso(26) y Morassi 2 casos(27). Los pacientes reportados en su mayoría comparten factores de riesgo vascular con la infección por SARS-CoV-2. Además en el contexto de la infección ocurren trastornos de coagulación, plaquetopenia y el síndrome de tormenta de citocinas los cuales también pueden ser factor de riesgo $(28,29)$ (Figura1).

Parkinsonismos, según la SEN reportó 1,1\%, en nuestra casuística fueron 2 casos, ambos de sexo masculino, 48años, diabéticos y obesos, con síndrome de distrés respiratorio del adulto severo, uno de ellos con RT-PCR positivo y otro con prueba rápida reactiva, con tomografía de tórax CO-RADS 5-6, ambos ingresaron a ventilación mecánica por 24 y 31 días. Al examen físico post ventilación mecánica presentaban facie inexpresiva, cuadro rígido acinético, sin temblor de reposo, sin piramidalismo. Tomografía cerebral sin contraste normal. Se han reportado investigaciones por otros virus con neurotropismo como durante la pandemia por Influenza H1N1 en la cual una de las formas de presentación eran los cuadros parkinsonianos (10).

- Encefalitis y meningoencefalitis (MEC): evaluamos 2 casos de encefalitis y 1 de meningoencefalitis viral asociada a COVID-19, ingresaron con SDRA severo, 1 con prueba rápida Ig $\mathrm{M} \mathrm{y} \mathrm{Ig} \mathrm{G} \mathrm{reactiva} \mathrm{y}$ 2RT-PCR positivos, recibieron aciclovir.

En los casos con encefalitis, paciente de sexo femenino, 77años, ingresó con 2 días de disnea y 1 día previo al ingreso a emergencia presentó sopor, hemiplejía izquierda y a los $3 \mathrm{~d}$ de hospitalizada entró en status convulsivo, líquido cefalorraquídeo (LCR) incoloro transparente, escasa pleocitosis con hiperproteinorraquia y glucosa normal. Resonancia de encéfalo compromiso cortico subcortical a nivel de región frontal derecha en FLAIR, pérdida de la sulcación por engrosamiento de circunvoluciones, sin realce meníngeo al contraste. Falleció a los 9 días (Figura 1). Otro paciente de sexo masculino, 53 años, ingresó con 4 días de enfermedad caracterizado por malestar general, dolor faríngeo, tos, fiebre, crisis convulsiva, tomografía cerebral sin contraste tenue hipodensidad frontal izquierda, LCR sin pleocitosis, con hiperproteinorraquia y glucosa normal, falleció a los 9 días.

El paciente con MEC viral de sexo masculino, 48 años, diabético, obeso, ingresó con un tiempo de enfermedad de 2 días por convulsiones y cefalea. 
Tomografía cerebral normal. LCR 120células $/ \mathrm{mm}^{3}$, $100 \%$ mononucleares, hiperproteinorraquia y glucosa normal. Alta a los 13días.

En la literatura mundial, los casos de encefalitis asociadas a COVID-19 son escasos o nulos. Sin embargo en algunos reportes se logró demostrar la presencia del virus en el LCR. Xiang quien publicó un caso de encefalitis en una paciente de 56 años de Wuhan, que fue diagnosticada de COVID-19 en enero de 2020, la paciente fue ingresada en una unidad de cuidados intensivos y presentó disminución del nivel de conciencia, por lo que se realizó una tomografía cerebral normal, el diagnóstico de encefalitis se confirmó al aislarse el SARS-CoV-2 en el líquido cefalorraquídeo mediante técnicas de secuenciación genómica(30). Moriguchi, describió un segundo caso de meningoencefalitis en un varón japonés de 24 años con síntomas de COVID-19, que presentó crisis epilépticas generalizadas y disminución del nivel de conciencia, detectó el SARS-CoV-2 en el líquido cefalorraquídeo mediante PCR-TR, el análisis del líquido cefalorraquídeo evidenció 12 células $/ \mu \mathrm{L}$ (10 mononucleares y dos polimorfonucleares), en la resonancia de encéfalo observaron áreas hiperintensas en el ventrículo lateral derecho, la región mesial del lóbulo temporal y el hipocampo, el paciente precisó ventilación mecánica invasiva debido a la neumonía y a las múltiples crisis generalizadas que presentó(7). El reporte de Zhou L et al., en el que señalan haber identificado el virus en LCR por secuenciación genética, es una descripción escueta que no brinda datos sobre el cuadro clínico, el tratamiento ni la evolución(31). Pilotto et al, describieron el caso de un sujeto de 60 años por lo demás sano con infección por SARS-CoV-2 pero solo anomalías respiratorias leves que desarrolló encefalopatía progresiva grave asociada con pleocitosis leve e hiperproteinorraquia, con resonancia magnética negativa, mientras que el EEG mostró ondas theta en las regiones anteriores del cerebro, los análisis de suero y LCR excluyeron otros trastornos infecciosos o autoinmunitarios conocidos, el paciente mejoró después del tratamiento con dosis altas de esteroides, lo que sugiere una afectación cerebral mediada por inflamación relacionada con la infección por SARS-CoV-2 (29). Las edades de estos casos fluctuaron entre 11 y 60 años, correspondiendo 5 casos a pacientes adultos, se ha descrito un predominio del sexo masculino(7,13,30-34). En los reportes referidos, sólo en el estudio de Duong, la paciente tenía obesidad y diabetes mellitus, en los otros reportes no se hace explícita la presencia o ausencia de algún factor de riesgo. En 3 reportes, los síntomas respiratorios fueron escasos, a diferencia de nuestros 3 casos con SDRA. En ninguno de nuestros casos fue factible RT-PCR para detección del virus SARS$\mathrm{CoV}-2$ en LCR, no disponible en nuestro hospital y tampoco en el Instituto Nacional de Salud (INS).

La mayoría de estudios de encefalitis aguda en el contexto de una infección por SARS-CoV-2 demostraron negatividad del PCR en LCR lo cual indicaría que no el mecanismo principal sería de tipo indirectos, inmunomediados (anticuerpos, citoquinas) producto de la respuesta inflamatoria e inmune sistémica y no a mecanismos directos de invasión sobre el encéfalo (35).

Las manifestaciones del SNP se dieron en 94 pacientes $(8,4 \%)$, destacando como síntomas las mialgias, anosmia y disgeusia, estas dos últimas similares a lo reportado por la SEN.

La mialgia, es el síntoma neurológico del SNP por afectación musculoesquelética más frecuente con 38 casos (3,4\%). Mao describió hasta 10,7\%, Wang en una serie retrospectiva de 138 casos en Zhongnan de Wuhan alcanzó 34,8\%, la SEN describe mialgia en 6 series con un porcentaje promedio del $27 \%$ (11).

La anosmia, estuvo presente en 35 pacientes $(3,1 \%)$, Mao describe un 5,1\%; Vaira de 320 pacientes reportó un 19,4\% de anosmia-ageusia(36). En la SEN alcanzó 19,6\% anosmia-hiposmia. En COVID-19, la anosmia típicamente no va acompañado de congestión nasal o rinitis. Es preciso mencionar que la anosmia no es específica de esta infección, puede ser secundaria a infección por influenza, con una incidencia estimada en Estados Unidos de 1 por cada 400 habitantes. Pero su aparición en esta coyuntura podría orientar al diagnóstico de COVID-19. Se conoce que las células de la mucosa olfatoria y respiratoria expresan el gen del receptor ACE2 lo cual tendría un papel en su patogénesis (11).

La ageusia/disgeusia se presentó en 32 pacientes $(8,8 \%)$, Mao reportó un 5,6\% de una serie de 214 pacientes.

Rabdomiólisis, se encontró 15 pacientes $(1,3 \%)$, Mao reportó daño muscular severo de 19\%(10). La SEN reportó rabdomiólisis en 2,1\% (11).

Las poliradiculoneuropatías agudas fueron 3 casos $(0,3 \%)$. El Síndrome de Guillain Barré (SGB) es un trastorno inmunomediado frecuentemente de aparición 
post infecciosa mediante el mecanismo de mimetismo molecular. Generalmente las infecciones se reportan 2-4 semanas antes del inicio de los síntomas neurológicos del SGB. La infección más común asociada con el desarrollo de SGB es la campilobacteriosis, también se han asociado infecciones virales como el citomegalovirus, el virus de Epstein-Barr, el virus de la inmunodeficiencia humana y el virus del Zika(3739). Coronavirus también han sido implicados como un desencadenante; en un estudio retrospectivo del brote de MERS-CoV, un paciente fue diagnosticado con encefalitis de Bickerstaff con SGB superpuesto, Tsai informó afectación neuromuscular en pacientes infectados con el SARS-CoV(5,39). La SEN reportó SGB en 7 casos (7.6\%). Toscano describió, 4 pacientes con SGB y 1 paciente con variante Miller Fisher después de COVID-19(8,40).

\section{CONCLUSIONES}

La pandemia originada por la infección por SARS$\mathrm{CoV}-2$, ha ocasionado una alta morbimortalidad a nivel mundial. Perú fue considerado por la Universidad Johns Hopkins, de Estados Unidos, como el primer país a nivel de latinoamérica con mayor número de muertes por millón de personas y no de los países con mayor cantidad de casos.

Este trabajo fue realizado con el propósito de describir las diferentes manifestaciones neurológicas asociadas al COVID-19 en nuestro centro hospitalario: el $69 \%$ eran mayores de 50 años, predominaba el sexo masculino, las comorbilidades más frecuentes fueron: HTA, DM, obesidad. Dentro de las manifestaciones del SNC predominaron la cefalea, encefalopatía, ACV y para el SNP mialgia, anosmia, ageusia, rabdomiólisis. La tasa de letalidad observada en los pacientes con infección por SARS-CoV-2 alcanzó el 28\% (314 fallecidos de 1122); la tasa de letalidad de los pacientes con manifestaciones neurológicas asociadas infección por SARS-CoV-alcanzó 32.7\% (116 fallecidos de 354) y con un OR de 1,4.

Hay que reconocer que el SARS-CoV-2 está muy extendido en nuestra población. Por lo tanto, una cierta proporción de pacientes con manifestaciones neurológicas en el contexto de la pandemia COVID-19 puede tener un mecanismo etiopatogénico distinto del SARS-CoV-2. Sin embargo, el personal de salud debe conocer que algunas de las manifestaciones neurológicas si podrían estar directamente asociadas a COVID -19, la cual es una enfermedad emergente, que cada día vamos descubriendo; así que debemos ser rápidos en nuestro enfoque para evitar diagnósticos erróneos, brindar un tratamiento oportuno y evitar una mayor morbimortalidad, como la encontrada en el presente estudio.

Consideraciones éticas: Se cumplieron los procedimientos y respetaron las normas éticas concordantes con la Declaración de Helsinki, los datos que identificaban a los pacientes fueron encriptados para proteger su confidencialidad y privacidad, los datos fueron obtenidos según el registro del sistema informático al egreso de los pacientes, por lo cual no fue posible obtener su consentimiento informado.

\section{Correspondencia}

Dra. Evelyn Vanessa Mariños Sánchez

Hospital Nacional Edgardo Rebagliati Martins

Av. Rebagliati 490 Jesús Maria

Teléfono: 511-265-4901 anexo: 15144

Correo electrónico: evelynmarinos1801@gmail.com

\section{REFERENCIAS BIBLIOGRAFICAS}

1. Huang C, Wang Y, Li X, Ren L, Zhao J, Hu Y, et al. Clinical features of patients infected with 2019 novel coronavirus in Wuhan, China. Lancet. 2020; 395(10223):497-506.

2. Lu R, Zhao X, Li J, Niu P, Yang B, Wu H, et al. Genomic characterisation and epidemiology of 2019 novel coronavirus: implications for virus origins and receptor binding. The Lancet. 2020;395(10224):56574.

3. ArcGIS Online. Coronavirus COVID-19 (2019nCoV). ArcGIS Online; 2020. [cited 2020 Sep 7]. Available from: https://www. arcgis.com/apps/opsdashboard/index.html\#/ bda7594740fd40299423467b48e9ecf6

4. Chen $\mathrm{X}$, Laurent S, Onur OA, Kleineberg NN, Fink GR, Schweitzer F, et al. A systematic review of neurological symptoms and complications of COVID-19. J Neurol. 2020;1: 1-11. doi: 10.1007/ s00415-020-10067-3

5. Kim J-E, Heo J-H, Kim H, Song S, Park S-S, Park $\mathrm{T}-\mathrm{H}$, et al. Neurological Complications during Treatment of Middle East Respiratory Syndrome. J Clin Neurol Seoul Korea. 2017;13(3):227-33.

6. Baig AM, Khaleeq A, Ali U, Syeda H. Evidence of the COVID-19 Virus Targeting the CNS: Tissue Distribution, Host-Virus Interaction, and Proposed Neurotropic Mechanisms. ACS Chem Neurosci. 2020;11(7):995-8.

7. Moriguchi T, Harii N, Goto J, Harada D, Sugawara H, Takamino J, et al. A first case of meningitis/ 
encephalitis associated with SARS-Coronavirus-2. Int J Infect Dis. 2020; 94:55-8.

8. Toscano G, Palmerini F, Ravaglia S, Ruiz L, Invernizzi P, Cuzzoni MG, et al. Guillain-Barré Syndrome Associated with SARS-CoV-2. N Engl J Med. 2020; 382(26):2574-2576. doi: 10.1056/ NEJMc2009191

9. Grau AJ, Buggle F, Becher H, Zimmermann E, Spiel $\mathrm{M}$, Fent T, et al. Recent bacterial and viral infection is a risk factor for cerebrovascular ischemia: clinical and biochemical studies. Neurology. 1998;50(1):196203.

10. Mao L, Jin H, Wang M, Hu Y, Chen S, He Q, et al. Neurologic Manifestations of Hospitalized Patients With Coronavirus Disease 2019 in Wuhan, China. JAMA Neurol. 2020;77(6):683.

11. Ezpeleta D, Azorín DG. Manual COVID-19 para el neurólogo general. Madrid: Ediciones SEN; 2020.p. 78.

12. Mariños E, Barreto-Acevedo E, Espino P. Accidente cerebrovascular isquémico asociado a COVID-19: primer reporte de casos en Perú. Rev Neuro-Psiquiatr. 2020; 83(2):127-33.

13. Barreto-Acevedo E, Mariños E, Espino P, Troncoso J, Urbina L, Valer N. Encefalitis aguda en pacientes COVID-19: primer reporte de casos en Perú. Rev Neuropsiquiatr. 2020; 83(2):116-22.

14. Helms J, Kremer S, Merdji H, Clere-Jehl R, Schenck M, Kummerlen C, et al. Neurologic Features in Severe SARS-CoV-2 Infection. N Engl J Med. 2020; 382(23):2268-70.

15. Zubair AS, McAlpine LS, Gardin T, Farhadian S, Kuruvilla DE, Spudich S. Neuropathogenesis and Neurologic Manifestations of the Coronaviruses in the Age of Coronavirus Disease 2019: A Review. JAMA Neurol. 2020; 77(8):1018-27.

16. Yaghi Shadi, Ishida Koto, Torres Jose, Mac Grory Brian, Raz Eytan, Humbert Kelley, et al. SARSCoV-2 and Stroke in a New York Healthcare System. Stroke. 2020; 51(7):2002-11.

17. Klok FA, Kruip MJHA, van der Meer NJM, Arbous MS, Gommers D, Kant KM, et al. Confirmation of the high cumulativeincidence of thrombotic complications in critically ill ICU patients with COVID-19: An updated analysis. Thromb Res. 2020; 191:148-50.

18. Lodigiani C, Iapichino G, Carenzo L, Cecconi M, Ferrazzi P, Sebastian T, et al. Venous and arterial thromboembolic complications in COVID-19 patients admitted to an academic hospital in Milan, Italy. Thromb Res. 2020;191:9-14.

19. Tan Y-K, Goh C, Leow AST, Tambyah PA, Ang A, Yap E-S, et al. COVID-19 and ischemic stroke: a systematic review and meta-summary of the literature. J Thromb Thrombolysis. 2020;50(3):587595. doi: 10.1007/s11239-020-02228-y

20. Oxley TJ, Mocco J, Majidi S, Kellner CP, Shoirah
H, Singh IP, et al. Large-Vessel Stroke as a Presenting Feature of Covid-19 in the Young. N Engl J Med. 2020; 382(20):e60. doi: 10.1056/NEJMc2009787

21. Gunasekaran K, Amoah K, Rajasurya V, Buscher MG. Stroke in a young COVID-19 patient. QJM Int J Med. 2020;113(8):573-4.

22. Umapathi T, Kor AC, Venketasubramanian N, Lim CCT, Pang BC, Yeo TT, et al. Large artery ischaemic stroke in severe acute respiratory syndrome (SARS). J Neurol. 2004; 251(10):1227-31.

23. Valderrama EV, Humbert K, Lord A, Frontera J, Yaghi S. Severe Acute Respiratory Syndrome Coronavirus 2 Infection and Ischemic Stroke. Stroke. 2020;51(7):e124-e127. doi: 10.1161/ STROKEAHA.120.030153

24. Boehme AK, Luna J, Kulick ER, Kamel H, Elkind MSV. Influenza-like illness as a trigger for ischemic stroke. Ann Clin Transl Neurol. 2018; 5(4):456-63.

25. Li Y, Li M, Wang M, Zhou Y, Chang J, Xian Y, et al. Acute cerebrovascular disease following COVID-19: a single center, retrospective, observational study. Stroke Vasc Neurol. 2020;5(3):279-284. doi: 10.1136/ svn-2020-000431

26. Sharifi-Razavi A, Karimi N, Rouhani N. COVID-19 and intracerebral haemorrhage: causative or coincidental? New Microbes New Infect. 2020;35: 100669.

27. Morassi M, Bagatto D, Cobelli M, D’Agostini S, Gigli GL, Bnà $C$, et al. Stroke in patients with SARS-CoV-2 infection: case series. J Neurol. 2020;267(8):218592.

28. Saavedra JM. Brain Angiotensin II: New Developments, Unanswered Questions and Therapeutic Opportunities. Cell Mol Neurobiol. 2005; 25(3):485-512.

29. Pilotto A, Odolini Si, Masciocchi S, Comelli A, Volonghi I, Gazzina S, et al. Steroid-responsive encephalitis in Covid-19 disease [Internet]. Ann Neurol. 2020;88(2):423-427. doi: 10.1002/ana.25783

30. Xiang P, Xu XM, Gao LL, Wang HZ, Xiong HF, Li RH, et al. First case of 2019 novel coronavirus disease with encephalitis. ChinaXiv 2020; T202003: 00015.Duong L, Xu P, Liu A. Meningoencephalitis without respiratory failure in a young female patient with COVID-19 infection in Downtown Los Angeles, early April 2020. Brain Behav Immun. 2020;87:33.

31. Zhou L, Zhang M, Wang J, Gao J. Sars-Cov-2: Underestimated damage to nervous system. Travel Med Infect Dis. 2020;36:101642.

32. Duong L, Xu P, Liu A. Meningoencephalitis without respiratory failure in a young female patient with COVID-19 infection in Downtown Los Angeles, early April 2020. Brain Behav Immun. 2020;87:33.

33. Ye M, Ren Y, Lv T. Encephalitis as a clinical manifestation of COVID-19. Brain Behav Immun. 2020;88:945-6. 
34. McAbee GN, Brosgol Y, Pavlakis S, Agha R, Gaffoor M. Encephalitis Associated with COVID-19 Infection in an 11-Year-Old Child. Pediatr Neurol. 2020;109:94.

35. Pezzini A, Padovani A. Lifting the mask on neurological manifestations of COVID-19. Nat Rev Neurol. 2020;16(11):636-44.

36. Vaira LA, Salzano G, Deiana G, De Riu G. Anosmia and Ageusia: Common Findings in COVID-19 Patients. The Laryngoscope. 2020;130(7):1787.

37. Yuki N, Hartung H-P. Guillain-Barré Syndrome. N Engl J Med. 2012; 366(24):2294-304.

38. Barbi L, Coelho AVC, Alencar LCA de, Crovella S. Prevalence of Guillain-Barré syndrome among Zika virus infected cases: a systematic review and meta-analysis. Braz J Infect Dis Off Publ Braz Soc Infect Dis. 2018;22(2):137-41.
39. Tsai L-K, Hsieh S-T, Chao C-C, Chen Y-C, Lin $\mathrm{Y}-\mathrm{H}$, Chang S-C, et al. Neuromuscular disorders in severe acute respiratory syndrome. Arch Neurol. 2004; 61(11):1669-73.

40. Zhao H, Shen D, Zhou H, Liu J, Chen S. GuillainBarré syndrome associated with SARS-CoV-2 infection: causality or coincidence? Lancet Neurol. 2020; 19(5):383-4.

Recibido: 05/09/2020

Aceptado: 08/12/2020 